\title{
The Students
}

Bonnie ACKer, Sarah Lawrence College

John A. Almstrom, University of British Columbia

HaROLD W. BorkowsKi, Colgate University

Marcia J. Carpenter, Colorado Woman's College

LaWrence S. Cooley, Colgate University

Marsha N. Chalmus Cunningham, Sarah Lawrence College

George W. Cooper, Holy Cross College

David J. H. Dean, Austin College

Cornelia Dellenbaugh, University of Pennsylvania

Kathy Sue Williams Doner, Stetson College

Mark Gerzon, Harvard University

Barbara S. Hancock, Radcliffe College

Thomas A. Hiat, Wabash College

JONATHAN JAY, University of Texas

VIDAR J. JORGENSEN, Harvard University 
Alan Kirshenbaum, University of Pennsylvania

Lorraine Benveniste Kirschenbaum, Alfred University

RichaRd LANDES, Harvard University

EDWARD M. LEBOW, Harvard University

Louise A. LeRner, Radcliffe College

ThOmas H. LeRner, Princeton University

John Mackie, JR., Claremont Men's College

Susan Meiselas, Sarah Lawrence College

William B. Michaelcheck, Southwestern College at Memphis

John V. Moller, Jr., Colgate University

LeWIS PeRry III, Harvard University

Jeffrey B. Peters, Harvard University

Ronaleen Ricciuti, Allegheny College

David P. Rubin, Lehigh University

Michael S. Siegal, Harvard University

Candace Slater, Pembroke College

JoHn H. ZAMmito, University of Texas 